\title{
Reducing the In-Vitro Electromagnetic Field Effect of Cellular Phones on Human DNA and the Intensity of Their Emitted Radiation
}

\author{
Maria Syldona, Ph.D. \\ Quantum Biology Research Lab, PO Box 428, Ridgway, CO. 81432 \\ quantumbio@ouraynet.com and \\ Formerly Assistant Professor at Dept. of Psychiatry, State University of NY at \\ Stony Brook, Medical School.
}

(Received April 8, 2007; Accepted with revision September 6, 2007)

\begin{abstract}
:
Several studies have demonstrated detrimental effects of cellular phone radiation on in-vitro biological systems. This article introduces a novel invitro method for demonstrating conformational changes in human DNA induced by a 5 minute exposure to cellular phone radiation emitted by an actual contemporary cellular phone. Dynamic changes in DNA conformation was determined in real-time by measuring the rate of DNA rewinding (in a spectrophotometer) following exposure to heat which causes the unwinding of the two strands of the helix. Cellular phone radiation produced a $40 \%$ increase in the rate of DNA rewinding. This effect was $95 \%$ attenuated when the experiment was repeated with the same cellular phone to which was attached a commercially available shielding disk shaped sheet containing a paramagnetic mineral. In a separate series of experiments the intensity of the cellular phone radiation was measured using an electromagnetic frequency spectrum analyzer. The intensity was reduced by approximately $50 \%$ in the presence of the shielding disk. Taken together these studies indicate the efficacy of a shielding disk to protect the body from cellular phone radiation.
\end{abstract}

Key Words: Cellular phones; EM radiation; DNA rewinding; DNA conformation; EM shielding device

\section{INTRODUCTION}

While there is growing evidence that many types of electromagnetic (EM) energies such as those involved in bone healing, wound healing and pain relief have beneficial effects on the body, other types of EM fields are harmful to the body. UV light for example is well known to cause skin cancer (melanoma) [1]. The science behind these phenomena is being studied within the emerging field of Bioelectromagnetics which now makes 
recommendations to the public regarding public safety. Original epidemiological studies demonstrated an increase incidence in respiratory tract cancer in US Navy personnel exposed to occupational microwave exposure [2]. Animal studies then demonstrated that microwaves and radiofrequency EM fields are also co-carcinogenic since they increase chemically induced skin cancer [3]. Cellular studies have verified that these carcinogenic effects are mediated by DNA since relatively weak EM fields increase DNA synthesis [4] and can modulate DNA repair [5]. Most definitive were studies demonstrating that electric fields could break or nick DNA strands [6], a phenomenon known to be associated with cancer [7]. Then these studies were extended to EM fields generated from common household devices like microwave ovens and computers which were also shown to be carcinogenic $[8,9]$. It wasn't long until cellular phones were added to the list when it was discovered that they increased specific oncogenes known to promote cancer $[8,9]$. Further elucidation of the role of DNA in EM field induced cancers came from Russian scientists who measured changed in the secondary structure (conformation) of DNA [10]. Since these carcinogenic effects of EM fields were exacerbated by repeated and prolonged exposure $[8,9]$ and occurred at relatively low EM field strengths, the safety of the public at large became a real concern.

By this point, scientists had figured the experimental resonance conditions required to reproduce these harmful biological effects [11]. Fortunately one of these experimental variables, the strength of the geomagnetic field at the time of the experiments, turned out to have a surprising effect. It was discovered (using nerve cells in tissue culture) that DC magnetic fields could interact with the offending radiofrequency fields and actually cancel out their detrimental effects [12]. The interference or neutralization of harmful bio-effects was subsequently confirmed and extended to include not only DC magnetic fields but all magnetic fields which are temporally incoherent and function as noise [13].

The present study measured the effects of cellular phone radiation on DNA conformation in-vitro and measured the intensity of the radiation emitted by cellular phones. DNA conformation was measured using classical biochemical renaturation techniques [14] as a measure of DNA rewinding. The intensity of cellular phone radiation was measured using a spectrum analyzer. The results demonstrate that cellular phone radiation speeds up DNA rewinding and that this effect is prevented when a commercially available shielding disk is placed in the presence of the cellular phone radiation. A second series of experiments demonstrated that this same shielding disk also reduced the intensity of radiation emitted from cellular phones.

\section{MATERIALS AND METHODS}

\section{Experimental Design}

In the first series of experiments, conformational changes in human DNA were measured in this study by monitoring rewinding in real-time. The procedure involved measuring the recovery of DNA after heat exposure known to unwind (denature) the two strands of the double-helix. Within 10 minutes after heating, the DNA quickly recovers by rewinding back to its original intact helical conformation [14] (Figure 1). The rewinding process can be monitored by measuring the absorption of light by chromophores in the DNA strands. Control experiments were conducted first in ambient EM fields. Then 
DNA rewinding was measured in the presence of the cellular phone. In the third experimental condition, DNA rewinding was measured with the same cellular phone with a commercially available shielding disk placed inside (or outside) the phone (Figure 2)

\section{DNA Rewinding}

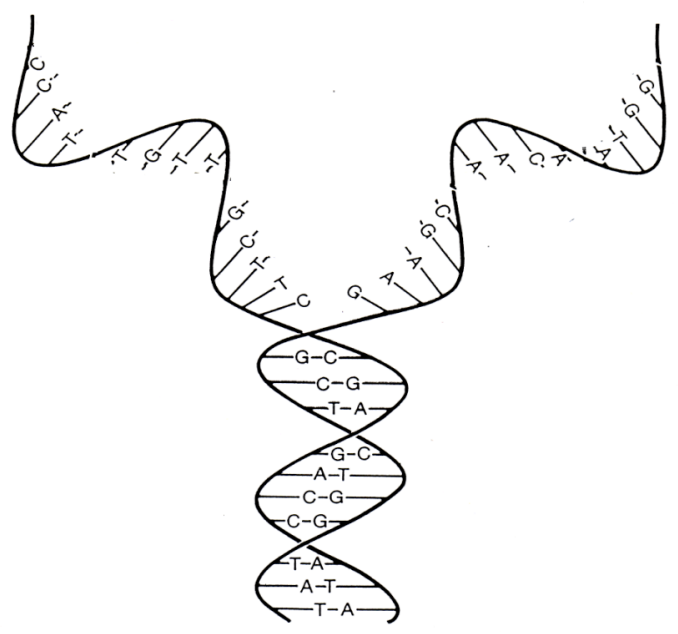

Figure 1: Graphical depiction of two strands of DNA partially unwound after heat $\left(80^{\circ} \mathrm{C}\right)$ treatment. As the DNA cools it spontaneously rewinds back to its original intact helical shape. Cellular phone radiation increases the rate of rewinding.

\section{The Neutralizing Disk}

A

\section{B}
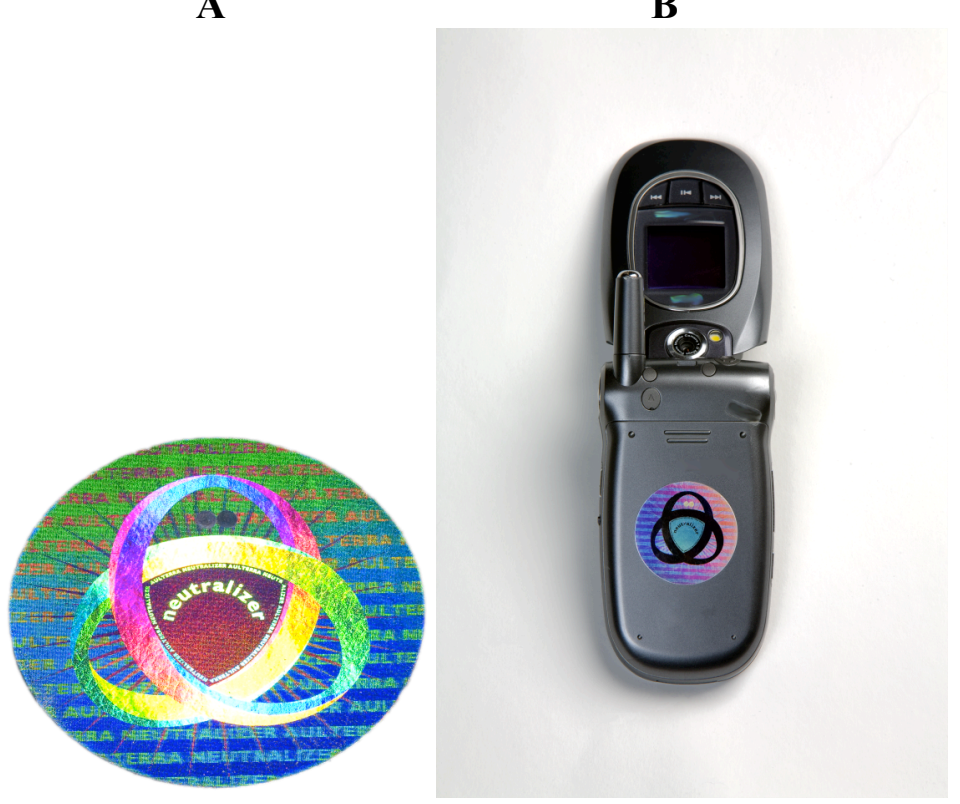

Figure 2: Figure A shows the Aulterra Neutralizer. Figure B shows the Verizon cellular phone with the neutralizing disk on the outside.

The shielding disk is composed of a sedimentary mineral rock powder obtained from a mine in Utah. Elemental analysis (Elemental Researach Inc., North Vancouver, BC, Canada) of the rock powder indicated the presence of typical non-metallic elements like 
silica, iron and titanium, as well as relatively high concentrations of the paramagnetic rare earth elements cerium and lanthanum. In addition, unusual crystalline structures were found. The rock powder is soft and brittle and is highly paramagnetic (Phil Callahan, personal communication). For the final product, the rock powder is milled to a $2 \mathrm{~m}$ particle size and activated by a propriety patented method involving the principles of homeopathy (US patent pending \# 60/820205). The final product, called the "Neutralizer" (which is commercially available through Aulterra International, Coeur D'Alene, ID, www.aulterra.com) is composed of three $0.1 \mathrm{~mm}$ layers of vinyl sheets $(3 \mathrm{~cm}$ diameter) each coated with the activated mineral using printing ink as a carrier.

A second series of experiments was conducted to determine the efficacy of the Aulterra Neutralizer in reducing the radiation emitted by a cellular phone. The intensity of the emitted radiation was measured using a spectrum analyzer.

\section{DNA Rewinding Assay (Series I)}

To determine the effect of cellular phone radiation on a model biological system, DNA rewinding was measured. A stock solution of human placental DNA (Sigma Chemical Co., St. Louis) in deionized water was further diluted to $0.03 \mathrm{mg} / \mathrm{ml}$ in deionized water and heated for 5 minutes at $80^{\circ} \mathrm{C}$. This treatment is typically used in in-vitro experiments to undwind the DNA helix. Under these standard conditions, the two strands of the helical DNA structure are known to unwind. Such cell-free experiments are typically done using water as the solvent because several salts present in buffer solutions are known to influence the conformation of DNA. Immediately after heat treatment the DNA was gently transferred to a quartz cuvette and measured in a spectrophotometer with the lid closed. For EM field exposure, a Verizon cellular phone (2004 model), in receiving-mode with no human voice transmitted, was placed face down on top of the cuvette inside the spectrophotometer (see Figure 3). The cellular phone remained in this position for the duration of the five minute experiment. The exact procedure was then repeated using the same cellular phone with the attached Aulterra Neutralizer.

Absorption of light at 260nm was measured using a UV-visible diode array spectrophotometer (Hewlett Packard 8451A) every 10 seconds over a five minute time period. As the DNA rewinds, its ability to absorb light decreases with time. Since rewinding measurements are taken in real-time, the DNA is being exposed to 10-second bursts of UV light (to measure rewinding), while it is being continuously exposed to cellular phone radiation (to influence rewinding). Since hours of high intensity UV-C radiation $(260 \mathrm{~nm})$ are required to damage DNA [14], it is unlikely that short bursts of low intensity UV-C from the spectrophotometer will affect DNA and influence its rewinding rate. The initial slope of the rewinding curve, typically used when studying kinetics of biochemical reactions, was calculated using IBM Excel software. All rewinding curves had a negative slope value. Slope values for the different experimental conditions were analyzed for statistical significance using a two sample t-test (assuming equal variance). A total of 12 control experiments, 14 cellular phone experiments and 22 neutralizedcellular phone experiments were used for these analyses. 
EXPERIMENTAL SETUP - SERIES 1

Spectrophotometer
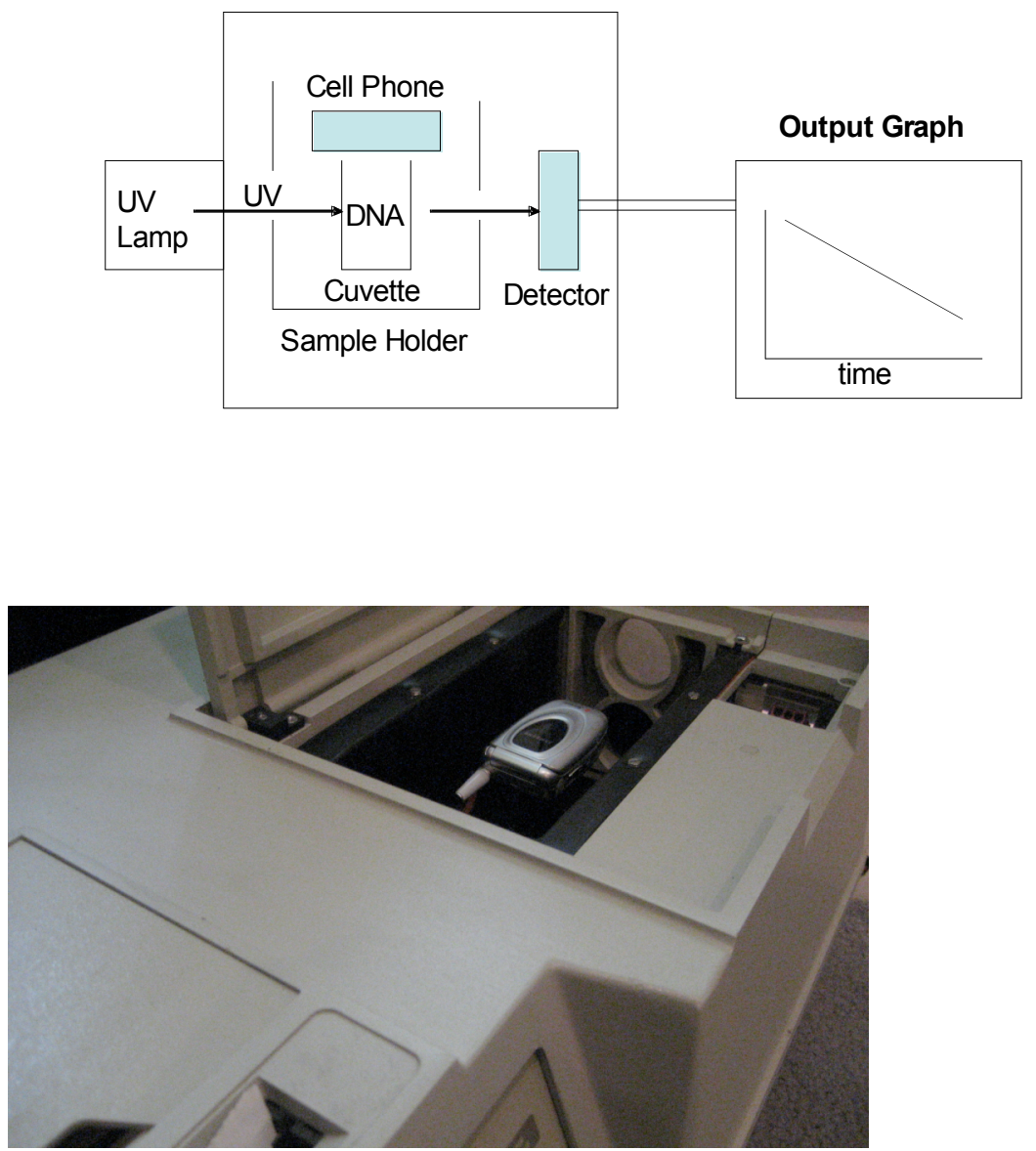

Figure 3: To measure rewinding, an aqueous sample of human DNA is placed in a cuvette inside a Hewlett Packard spectrophotometer which measures the absorption of UV light by the sample. Light absorption is measured every 10 seconds when the DNA is spontaneously rewinding. Note the position of the cellular phone whose radiated EM field influences the rewinding process of the DNA inside the cuvette underneath the cellular phone.

\section{Measurement of Cellular Phone Radiation (Series II)}

This series of experiments was designed to test the ability of the Aulterra Neutralizer to reduce the intensity of radiation emitted by a cellular phone. The radiation emitted by the same cellular phone used in Series I was measured by placing a cellular phone antennae (telescope variety from Radio Shack) on top of a cellular phone while receiving an active transmission. Such antennae are tuned to the specific frequencies emitted by cellular phones. For some experiments the phone was receiving a repeating, pre-recorded weather channel transmission. The signal detected by the antenna was then transferred to a spectrum analyzer (Agilent ESA-E series with FM modulation) (Figure 4). Six separate 
analyzer graphs, plotting intensity of emitted radiation vs frequency, were generated from the cellular phone and six additional graphs were generated using the same phone with the Aulterra Neutralizer attached. Representative graphs are presented here for each of the experimental conditions. To allow direct comparison of graphs with and without the Neutralizer, these graphs have the same Dbm intensity scale (y-axis).

EXPERIMENTAL SETUP-SERIES 2

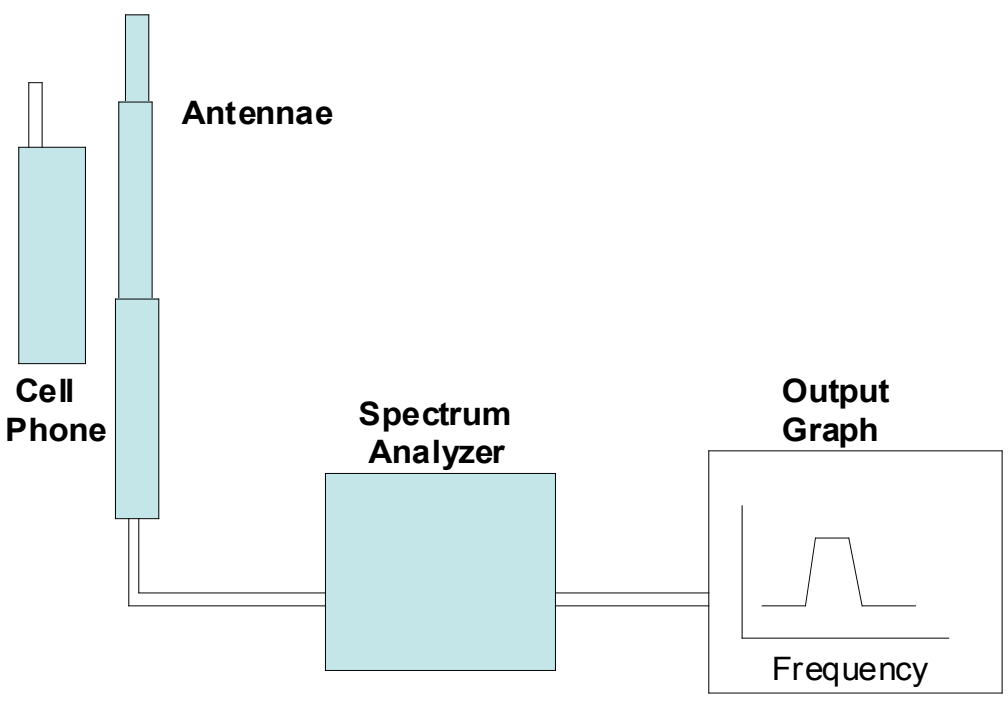

Figure 4: The intensity of EM radiation emitted by a cellular phone is measured by placing the phone immediately adjacent $(1.5 \mathrm{~cm})$ to a specially designed radio antennae which is connected to a spectrum analyzer. Such an antennae is commonly used to measure cellular phone radiation at short distances. The spectrum analyzer with FM demodulation was made by Agilent Technologies (San Francisco, CA) and measures frequencies from $9 \mathrm{kHz}$ to $3 \mathrm{GHz}$ with a sensitivity of $0.4 \mathrm{~dB}$. The trace recordings of the cellular phone signal is stored and printed out from the device's memory. Peak height values were measured accurately from the printout using a ruler.

\section{RESULTS}

\section{DNA Rewinding}

Figure 5 shows a typical initial DNA rewinding curve over several minutes. The light grey irregular line is a plot of the raw absorption data collected by the spectrophotometer. The solid black line is the computer generated best-fit calculation of the slope.

\section{Raw Spectrophotometric Data of DNA Rewinding}




\section{Control}

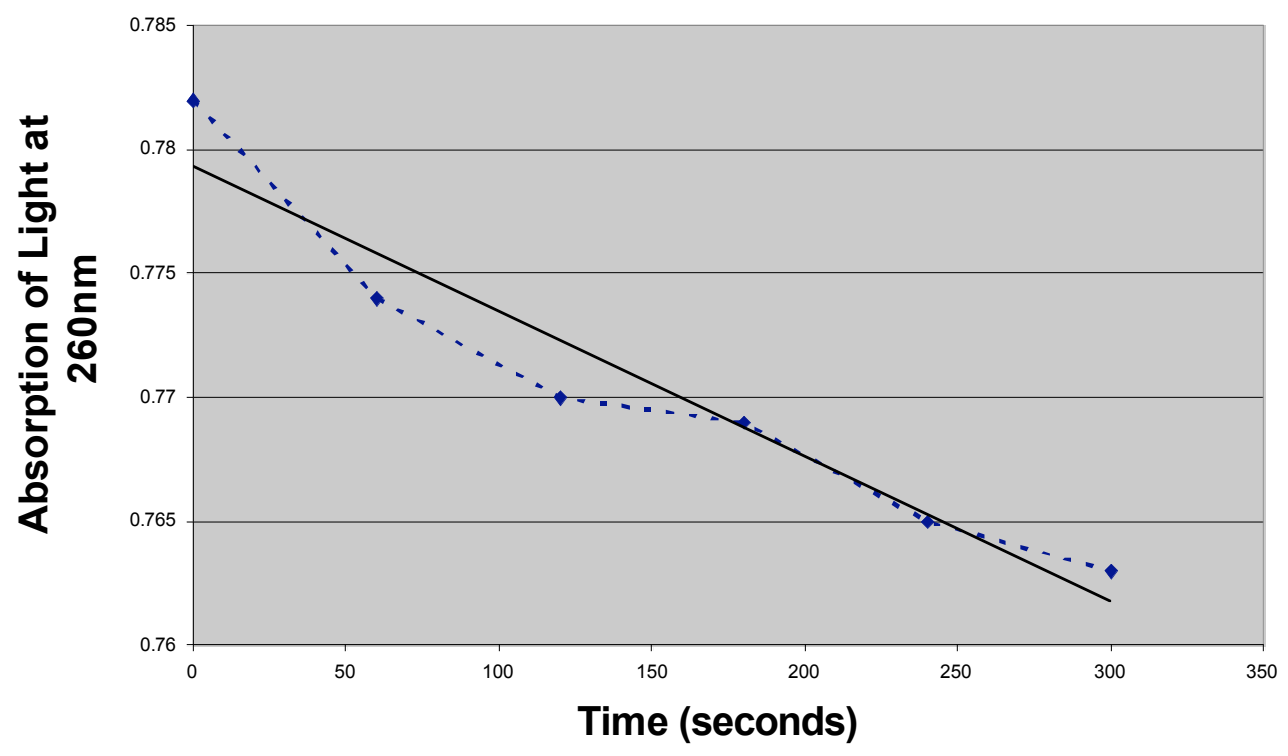

Figure 5: Hewlett Packard spectrophotometer readings of an aqueous solution of human placental DNA rewinding immediately following heat treatment in control conditions. Solid black line is computer generated best fit analysis used to calculate the slope (absorption/time) which is used as the rate of rewinding. The slope of this line is increased in the presence of cellular phone radiation.

\section{Electromagnetic Fields from Cellular Phones Effect DNA Rewinding}

The results presented in Table 1 below indicate the effect of cellular phone radiation on DNA rewinding after heat shock. In the absence of cellular phone radiation (control experiments), the average slope over all 12 experiments was $-0.41 \pm 0.065$. In the presence of EM fields from the cellular phone, the slope had an average value of $-0.56 \pm$ 0.056 over all 14 experiments. A more negative value for the slope reflects a faster rewinding rate following heat shock. Therefore, the EM field from the cellular phone produced a $40 \%$ increase (relative to control values) in the rewinding rate. The magnitude of the effect varied from $20-58 \%$ depending on the experiment This effect of the cellular phone radiation is highly statistically significant compared to the untreated control $(\mathrm{p}<0.0001)$.

\section{Aulterra Neutralizer Attenuates Cellular Phone Induced Effects on DNA}

The results in Table 1 indicate that the cellular phone with an attached Neutralizer produced an average slope of $-0.43 \pm 0.11$ for all 22 experiments. This average value is not significantly different than the control value indicating that the effect of the cellular phone radiation on rewinding is completely reversed in the presence of the Neutralizer. When examining the data from the individual experiments (Figure 6), however, it is 
apparent that on some occasions the cellular phone radiation is not neutralized, eg. experient number 2, 3, 4, 10 and 13 . This indicates that for these particular treatment sessions, on a given day, resonance conditions were not met (see discussion).

\section{Raw Slope Values for DNA Rewinding}

\begin{tabular}{|l|c|c|c|c|c|}
\hline & Slope & SD & \% Change & n & p \\
\hline \hline Control & -0.41 & 0.065 & & 12 & \\
\hline Cellular Phone (CP) & -0.559 & 0.056 & +40 & 14 & $<0.0001$ \\
\hline CP +Neutralizer & -0.43 & 0.115 & +5 & 22 & NS \\
\hline
\end{tabular}

Table 1: Average rewinding slope values for each of the three experimental conditions calculated from computer-generated absorption curves obtained using a Hewlett Packard spectrophotometer. " $n$ " indicates the total number of separate experiments tested under each condition. SD refers to the standard deviation. Statistical significance, $p$, is calculated with respect to control values using t-tests on raw slope values. NS is not significant.

Effect of Cellular Phone with Attached Shielding Disk on DNA Rewinding

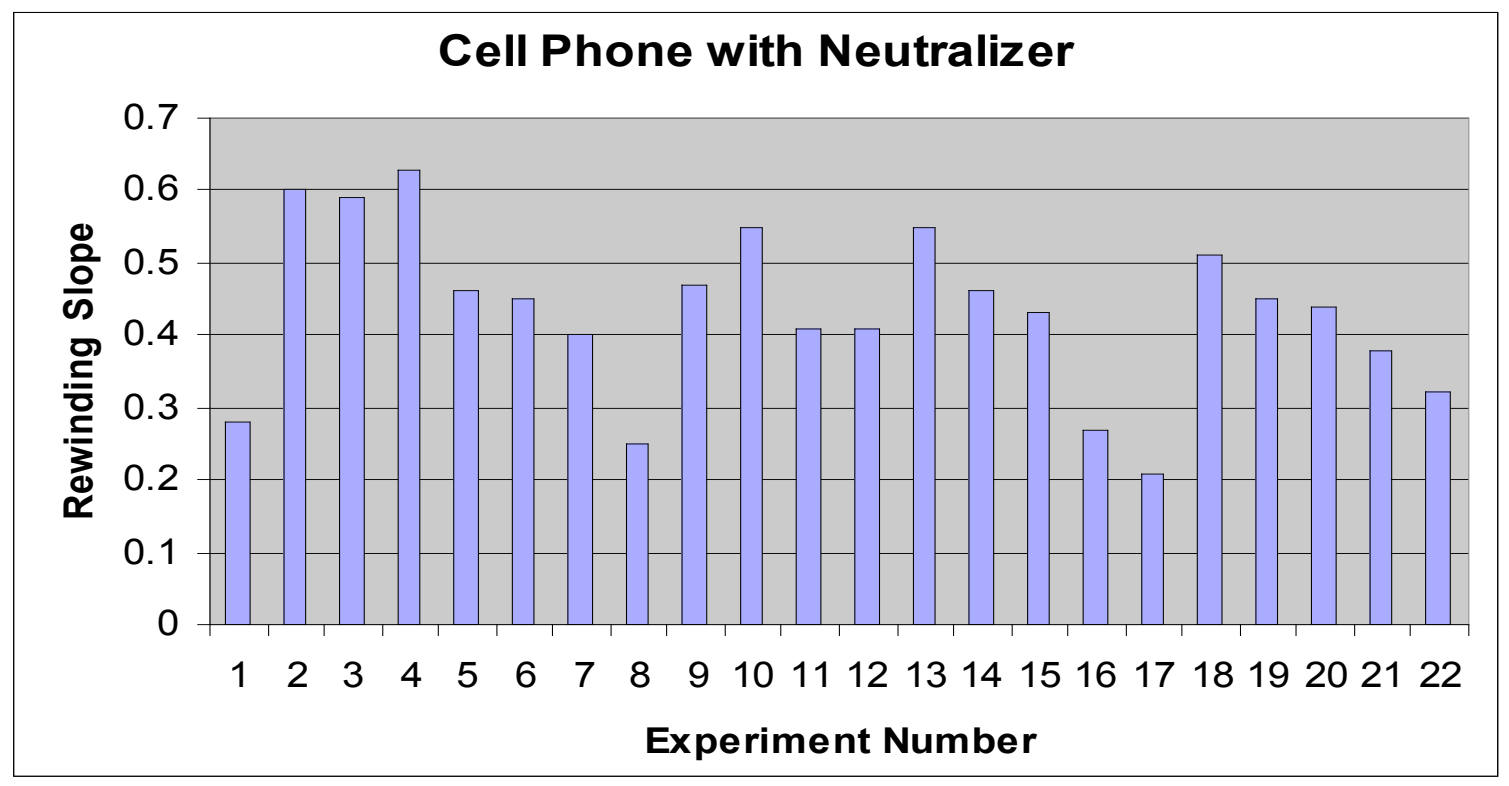

Figure 6: Variation of rewinding slope values for each of 22 trials whose averages are presented in Table 1. Rewinding slope values were calculated from computer-generated absorption curves obtained using a Hewlett Packard spectrophotometer. DNA samples were treated with a cellular phone with the EM shielding device attached.

\section{Aulterra Neutralizer Reduces Cellular Phone Radiation}

Several discrete frequencies emitted from the cellular phone could be measured with the spectrum analyzer and their intensities were significantly above background fluctuations. 
Large discrete peaks occurred at specific frequencies, eg 54, 72 and $94 \mathrm{MHz}$. In the presence of the Aulterra Neutralizer a reduction in the intensity of all peaks was observed and the peak at $94 \mathrm{MHz}$ was studied quantitatively. Over six experiments, the average intensity of the $94 \mathrm{MHz}$ peak was $36.4 \pm 1.3 \mathrm{dBm}$. With the Neutralizer this peak decreased in size to a value of $17.6 \pm 2.5 \mathrm{dBm}$. This $52 \%$ (2-fold) reduction is highly statistically significant $(\mathrm{p}<0.0001)$ (Figure 7$)$. Since the two smaller side peaks were absent when measuring a signal from an open channel with no human voice transmission, they are likely due to human voice modulation of the cellular phone signal. In the presence of the Neutralizer, both side peaks were completely eliminated. These results confirm a previous study demonstrating that another commercially available shielding device, which also contained rare earth elements, effectively reduced the EM fields emitted by video display terminals associated with computer monitors [15].

\section{Spectrum Analyzer Measurements of Cellular Phone Radiation}

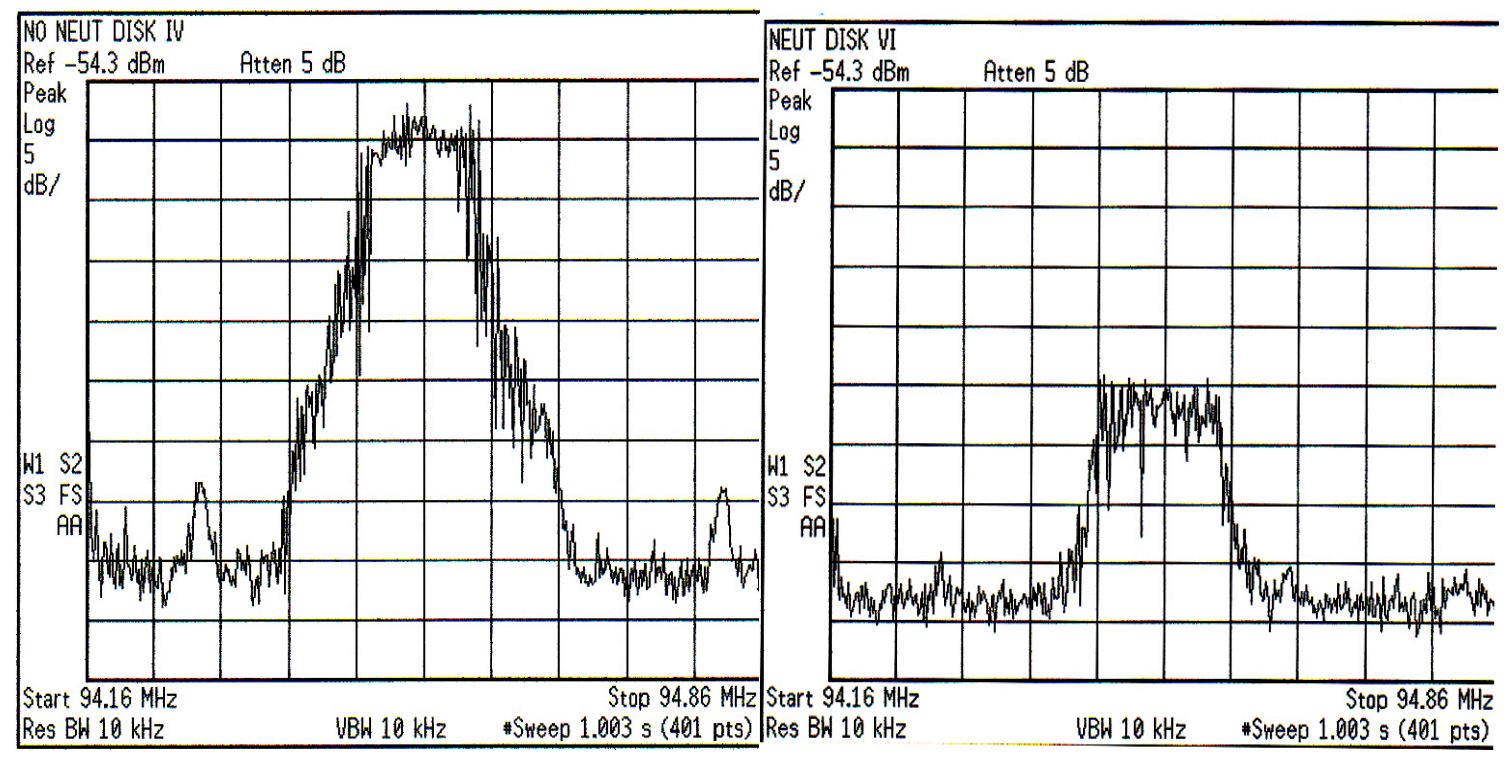

Figure 7: Typical spectrum analyzer traces centering at $94.16 \mathrm{MHz}$. Peak heights are related to the intensity of cellular phones radiation. Left trace is a recording from a normal cellular phone and the right trace is recorded from the same cellular phone with the shielding device attached.

\section{DISCUSSION}

Experiments performed in Series I utilize a classical property of DNA, rewinding, as a new model system for studying in-vitro effects of cellular phone radiation. The results indicate that cellular phone radiation significantly speeds up the rate of rewinding. This effect is highly reproducible in the 14 independent experiments with a magnitude that varied between 20 and 58\%. Data from the present study support Blank's hypothesis that DNA itself can act as a target for EM fields [16]. This hypothesis contradicts years of previous Bioelectromagnetics data indicating that the primary target for EM fields is the plasma membrane and/or the ions that bind to various protein receptors within the plasma 
membrane. However, several studies support the DNA antennae theory and have shown that various functional properties of DNA including DNA synthesis [4] and repair [5] are altered by EM fields.

These direct effects on DNA maybe mediated by its conformational state. Original studies demonstrated electromagnetic field effects on DNA conformational states [10]. The conformation, or secondary structure of all biomolecules is intimately linked to their functional properties [17]. Conformational states are in turn controlled by hydrogen bonds which hold together the folded secondary structure of biomolecules [18]. DNA winding experiments in-vitro, like those utilized in the present study, can be considered an indirect measurement of hydrogen bond formation. In the present study it was shown that cellular phone radiation speeds up DNA rewinding suggesting there is an increase in hydrogen bond formation. If cellular phones radiation is eventually shown to have a direct effect on hydrogen bonds in general, the implications for biology could be profound. In support of this hypothesis, it has been observed that radiation from cellular phones alters the conformation of human chromatin [19].

Since the implications for public health are obvious, methods to reduce the harmful effects of cellular phone radiation are currently being sought. New technologies have emerged utilizing a novel concept that one type of EM field can reverse or neutralize the biological effects produced by another type of EM field. This was first demonstrated using DC magnetic fields capable of neutralizing the effect of a pulsed magnetic field on neurite outgrowth in neuronal cellular studies [12]. Yet another similar phenomenon was demonstrated using incoherent noise fields to neutralize magnetic field effects on ornithine decarboxylase induction [13]. At least one example of how this technology can be applied to cellular phone protection was recently demonstrated in a study using a commercially available aluminum EM shield. In this study Goodman [20] used the number of fruit fly offspring, as well as some biochemical markers, as a model system and showed that cellular phone radiation increased the number of offspring and increased levels of transcription factors which are known to activate DNA synthesis. This effect was reversed when a commercially available aluminum shield was added to the cellular phone. These results confirm earlier work by Omura $[8,9]$ indicating that grounding electronic devices with a metal sheet reduced (or eliminated) their radiated EM fields.

The present study demonstrates a similar phenomenon as in the Goodman study, but utilizes a different model system. Here the cellular phone induced changes in DNA rewinding are completely reversed when an Aulterra Neutralizer is added to the cellular phone. This effect is seen $77 \%$ of the time (see Figure 6) yielding an over-all statistically significant effect. The fact that this effect is not seen in every experiment is interesting and suggests that resonance conditions are not met in every experiment. In order to produce biological effects, resonance between an EM field and a biological system is required. So far resonance conditions have been characterized in terms of narrow "windows" where biological effects are observed only within specific and narrow frequency and amplitude ranges of the EM field [11]. In the present case, resonance 
conditions require a complex interaction between the EM field from the cellular phone, the EM field generated from the Neutralizer disk itself (since it is paramagnetic) and the DNA molecule. In other similar systems (described above), where two EM fields interact, the strength of and orientation between the two fields is critical in order to obtain resonance conditions.

In the present experiment off-resonance conditions occur only $23 \%$ of the time. Experiments can be designed in such a way that off-resonance conditions occur frequently enough to conclude cellular phone radiation produces no damaging effects. Many of the scientific studies reported in the literature utilize such experimental designs. Redesigning such experiments can produce on-resonance conditions so that cellular phone effects can be measured. In real-world situations consumers are chronically exposed to cellular phone radiation which at times is either on or off-resonance.

Preliminary experiments indicate that the Aulterra Neutralizer contains a highly paramagnetic mineral (R. Roy, personal communication). It is therefore conceivable that a magnetoresistance response (Meissner effect) is induced when the Neutralizer is in the presence of the magnetic fields generated from cellular phones. Thus paramagnetic shielding (in addition to ferromagnetic shielding) is a likely explanation for the ability of the Neutralizer to reverse the effects of cellular phone radiation on DNA rewinding observed in this study. Ferromagnetic transduction has been previously proposed to mediate biological effects of cellular phones because of their resonance with the biological mineral magnetite [21].

The reversal of DNA rewinding effects observed in Series I of the present study is further supported by the results obtained by measuring the intensity of cellular phone radiation using a spectrum analyzer (Series II). Specific absorption rates are typically used to quantify the intensity of cellular phone radiation, which in turn is related to the severity of the biological damage and therefore public safety. The results of the present study clearly indicate that the intensity of the radiation emitted from cellular phones is reduced by approximately $50 \%$ in the presence of the Aulterra Neutralizer. In all experiments the Neutralizer was effective across the entire radio and microwave spectrum of radiation emitted by these devices. This neutralizing effect was also independent of the various experimental conditions, ie. the presence of voice patterns or EM signals from nearby electronic devices.

When the spectrum analyzer data is taken in conjunction with the DNA data, we have a strong case for concluding that the Aulterra Neutralizer is capable of protecting the body from cellular phone radiation. Although the bio-protective effect may be a result of reducing the intensity of the cellular phone radiation, it is also possible that a weak EM field generated by the Neutralizer could have a direct stabilizing effect on the DNA. Therefore, the Aulterra Neutralizer appears to protect the body by both a biological and a physical mechanism. This is the first report of any commercially available cellular phone shielding device capable of such a dual action. 


\section{CONCLUSION}

The changes in DNA conformation induced by cellular phones, as measured in this study, support previous studies demonstrating that DNA does in fact directly interact with cellular phone radiation. The in-vitro effect produced by such radiation was $95 \%$ reversed when a commercially available shielding disk is attached to a cellular phone. This finding, when taken together with experiments demonstrating that the shielding device also reduced the intensity of cellular phone radiation, supports the conclusion that the Aulterra Neutralizer inhibits the effects of cellular phone radiation on DNA in-vitro.

\section{ACKNOWLEDGMENTS}

Michael Manning, formerly of Temple University, for help in spectrum analyzer experiments, Dr. Rein for technical scientific advice without whom this research could not be completed and Kim Dandurand of Aulterra International for inspiration and support.

\section{REFERENCES}

1. Stenback, F. Species-specific neoplastic progression by ultraviolet light on the skin of rats, guinea pigs, hamsters and mice. Oncology. Vol 31, pp.209-225, 1975.

2. Robinette, C.D., Silverman, C., Jablon, S., Effects upon health of occupational exposure to microwave radiation. American Journal of Epidemiology. Vol 112, pp. 39$53,1980$.

3. Szmigielski, S., Szudzinski, A., Pietraszek, A., Bielec, M., Janiak, M., Wrembel, J.K., Accelerated development of spontaneous and benzopyrene-induced skin cancer in mice exposed to $2450 \mathrm{MHz}$ microwave radiation. Bioelectromagnetics. Vol 3, pp. 179-191, 1982.

4. Liboff, A.R., Williams, T. Jr., Strong, D.M., Wistar, R. Jr., Time-varying magnetic fields: effect on DNA synthesis. Science. Vol 223, pp. 818-820, 1984.

5. Meltz, M.L., Walker, K.A., Erwin, D.N., Radiofrequency radiation exposure of mammalian cells during UV-induced DNA repair synthesis. Radiation Research. Vol 110, pp. 255-266, 1987.

6. Tamiya, E., Nakajima, Y., Kamioka, H., Suzuki, M., Karube, I. DNA cleavage based on high voltage electric pulse. FEBS Letters. Vol 34, pp 357-61, 1988.

7. Kitchin, K.T., Brown, J.L., Biochemical studies of promoters of carcinogenesis in rat liver. Teratogenesis, Carcinogenesis and Mutagenesis. Vol. 9, pp. 273-85, 1989.

8. Omura, Y., Losco, M., Omura A.K., Yamamoto, S., Ishikawa, H., Takeshige C., Shimotsuura, Y., Muteki, T., Chronic or intractable medical problems associated with 
prolonged exposure to unsuspected harmful environmental electric, magnetic or electro-magnetic fields radiating in the bedroom or workplace and their exacerbation by intake of harmful light and heavy metals from common sources. Acupuncture and Electro-therapeutics Research, the International Journal. Vol 16, pp143-77, 1991.

9. Omura, Y., and Losco M., Electro-magnetic fields in the home environment (color TV, computer monitor, microwave oven, cellular phone, etc) as potential contributing factors for the induction of oncogene $\mathrm{C}$-fos $\mathrm{Ab} 1$, oncogene $\mathrm{C}$-fos $\mathrm{Ab} 2$, integrin $\alpha 5 \beta 1$ and the development of cancer, as well as effects of microwave on amino acid composition of food and living human brain. Acupuncture and Electrotherapeutics Research, the International Journal. Vol 18, pp.33-73, 1993.

10. Semin, Iu.A., Changes in secondary structure of DNA under the influence of electromagnetic fields. Radiation Biology and Radioecology. Vol 35, pp. 36-41, 1995

11. Blackman, C.F., Blanchard, J.P., Benane, S.G., House, D.E., The ion parametric resonance model predicts magnetic field parameters that affect nerve cells. FASEB Journal Vol 9, pp.547-551, 1995.

12. Blackman, C.F., Blanchard, J.P., Benane, S.G., House, D.E., Effect of AC and DC magnetic field orientation on nerve cells. Biochemical Biophysical Research Communications. Vol 220, pp. 807-811, 1996.

13. Farrell, J.M., Barber, M., Krause, D., Litovitz, T.A., The superposition of a temporally incoherent magnetic field inhibits $60 \mathrm{~Hz}$-induced changes in the ODC activity of developing chick embryos. Bioelectromagnetics Journal. Vol 19, pp. 53$56,1998$.

14. Marmur, J., and Doty, P., Thermal renaturation of DNA. Journal of Molecular Biology. Vol 3, pp. 585-594, 1961.

15. Sisto R, Casciardi S, Giliberti C, Moleti A. Electromagnetic radiation from VDT units: study of the effectiveness of an active shielding device. Journal of the American Industrial Hygiene Association. Vol 60, pp. 111-115, 1999.

16. Blank, M., and Goodman, R., Electromagnetic fields may act directly on DNA. Journal of Cellular Biochemistry. Vol 75, pp. 369-374, 1999.

17. Ninaber, A., Goodfellow, J.M., DNA conformation and dynamics. Radiation and Environmental Biophysics. Vol 38, pp. 23-29, 1999.

18. Takano, K., Yamagata, Y., Funahashi, J., Hioki, Y., Kuramitsu, S., Yutani, K., Contribution of intra- and intermolecular hydrogen bonds to the conformational stability of human lysozyme. Biochemistry. Vol 38, pp. 12698-12708, 1999. 
19. Belyaev, I.Y., Alipov, E.D., Frequency-dependent effects of ELF magnetic field on chromatin conformation in Escherichia coli cells and human lymphocytes.

Biochimica Biophysica Acta. Vol 1526, pp. 269-76, 2001.

20. Goodman, R., Lin, H., Ye, L., Weisbrot D. EM field-induced markers of delineators of interaction mechanism. Proceedings of the Bioelectromagnetics Society. Quebec City, Canada. June, 2002.

21. Cranfield, C., Wieser, H.G., Al Madan, J., Dobson, J., Preliminary evaluation of nanoscale biogenic magnetite-based ferromagnetic transduction mechanisms for wireless phone bioeffects. IEEE Transactions of Nanobioscience. Vol 2, pp. 40-43, 2003. 